\title{
Boundary effects on image reconstruction in photoacoustic tomography
}

Xinmai Yang, Lihong V. Wang

Xinmai Yang, Lihong V. Wang, "Boundary effects on image reconstruction in photoacoustic tomography," Proc. SPIE 6437, Photons Plus Ultrasound: Imaging and Sensing 2007: The Eighth Conference on Biomedical Thermoacoustics, Optoacoustics, and Acousto-optics, 64370W (13 February 2007); doi: 10.1117/12.698360

SPIE. Event: SPIE BiOS, 2007, San Jose, California, United States 


\title{
Boundary effects on image reconstruction in photoacoustic tomography
}

\author{
Xinmai Yang and Lihong V. Wang \\ Optical Imaging Laboratory, Department of Biomedical Engineering, Washington University in St. \\ Louis, St. Louis, Missouri 63130
}

\begin{abstract}
Exact photoacoustic tomography requires scanning over a $4 \pi$ solid angle in $3 \mathrm{D}$. The ultrasound detection window, however, is often limited, which makes a full scan impossible. For example, when a boundary lies closely to an object, the scanning region can cover only less than $4 \pi$ in 3D. Because of incomplete information, the resolution, SNR, and fidelity of the resulting image deteriorate. Boundaries, however, can be used to our advantage; we proposed postprocessing algorithms in image reconstruction to make partially scanned data complete. Here, we show the efficacy of the post-processing algorithms with both numerical and experimental results. Indeed, the algorithms can improve the resolution, SNR, and fidelity.
\end{abstract}

Keywords: photoacoustic tomography, boundary condition, image reconstruction

\section{INTRODUCTION}

Photoacoustic tomography (PAT) is a hybrid imaging technique. This technique combines the good spatial resolution of ultrasound and the good contrast of optical absorption for deep imaging in biological tissues. Compared to pure ultrasound imaging, which depends on the mismatch of mechanical properties in biological tissues, PAT relies on the mismatch of optical absorptions in biological tissues. The mismatch of optical absorptions provides much larger contrast than that of the mechanical properties. On the other hand, pure optical imaging suffers from strong light scattering by biological tissues. As a result, pure optical imaging can only be used for shallow imaging depth or provides low spatial resolution. However, the detected signals in PAT are ultrasound signals, which are very weakly scattered by biological tissues compared with optical signals; therefore PAT retains the good spatial resolution. Moreover, optical absorption is highly sensitive to biochemical molecules, such as oxygenated and deoxygenated hemoglobins, and can thus provide functional imaging.

The field of PAT has experienced considerable growth in the past few years. Until now, however, a boundary-free infinite acoustic medium has been assumed in all of the exact reconstruction algorithms for PAT [1]-[6]. In practice, a boundary may present in a certain imaging configurations. The presence of the boundary condition in the scanning region can limit the position of the detector and make a complete scan impossible. To obtain good image quality, the boundary condition has to be considered in the post-processing. A post-processing algorithm has been proposed to make partially scanned data complete. In this paper, we show the efficacy of the post-processing algorithms with both numerical and experimental results. In the following section, we review the photoacoustic equation and the reconstruction algorithm for PAT, then the boundary conditions which need to be take into account in the imaging processing. The numerical and experimental results are followed.

\section{PHOTOACOUSTIC EQUATION}

When a pressure rise is initiated by laser heating, acoustic waves develop and begin to propagate [7]. The acoustic wave generation and propagation in an inviscid medium is described by

Photons Plus Ultrasound: Imaging and Sensing 2007: The Eighth Conference on Biomedical Thermoacoustics, Optoacoustics, and Acousto-optics, edited by Alexander A. Oraevsky, Lihong V. Wang,

Proc. of SPIE Vol. 6437, 64370W, (2007) · 1605-7422/07/\$18 - doi: 10.1117/12.698360 


$$
\left(\nabla^{2}-\frac{1}{v_{s}^{2}} \frac{\partial^{2}}{\partial t^{2}}\right) p(\vec{r}, t)=-\frac{\beta}{\kappa v_{s}^{2}} \frac{\partial^{2} T(\vec{r}, t)}{\partial t^{2}},
$$

where $p(\vec{r}, t)$ is the acoustic pressure at location $\vec{r}$ and time $t ; T$ is the temperature rise; $v_{s}$ is the speed of sound; $\kappa$ is the isothermal compressibility; and $\beta$ is the thermal coefficient of volume expansion. The isothermal compressibility $\kappa$ can be expressed as

$$
\kappa=\frac{C_{P}}{\rho v_{s}^{2} C_{V}}
$$

where $\rho$ is the mass density; and $C_{P}$ and $C_{V}$ are the specific heat capacities at constant pressure and volume, respectively. The left-hand side of Eq.

(1) describes the acoustic wave propagation, while the right-hand side represents the source term.

In thermal confinement, the thermal equation becomes

$$
\rho C_{V} \frac{\partial T(\vec{r}, t)}{\partial t}=H(\vec{r}, t)
$$

where $H(\vec{r}, t)$ is the heating function defined as the thermal energy produced in tissue by the laser light per unit time and volume. The heating function is related to the optical energy deposition $Q_{e}$ by $Q_{e}=\int H d t$ and to the optical fluence rate $\Phi$ by $H=\mu_{a} \Phi$ if all of the absorbed optical energy is converted to heat. Substituting Eq. (3) into Eq. (1), we obtain the following less general photoacoustic equation, which is valid in thermal confinement only:

$$
\left(\nabla^{2}-\frac{1}{v_{s}^{2}} \frac{\partial^{2}}{\partial t^{2}}\right) p(\vec{r}, t)=-\frac{\beta}{C_{P}} \frac{\partial H}{\partial t} .
$$

Here, the source term is expressed as the first derivative of the heating function $H$ with respect to time $t$. This means that constant heating does not produce a pressure wave; only a change in heating does.

$$
p(\vec{r}, t)=\frac{1}{4 \pi v_{s}^{2}} \frac{\partial}{\partial t}\left[\frac{1}{v_{s} t} \int d \vec{r}^{\prime} p_{0}\left(\vec{r}^{\prime}\right) \delta\left(t-\frac{\left|\vec{r}-\vec{r}^{\prime}\right|}{v_{s}}\right)\right],
$$

which can be used to derive the response to delta heating of an arbitrary object. In PAT, Eq. (5) is inverted for image reconstruction.

The initial pressure can be reconstructed by the following back-projection formula:[8]

$$
p_{0}^{(b)}(\vec{r})=\frac{1}{\pi} \int_{-\infty}^{+\infty} d k \int_{S} d S \widetilde{p}^{*}\left(\vec{r}_{0}, k\right)\left[\hat{n}_{0}^{s} \cdot \nabla_{0} \widetilde{G}_{k}^{(o u t)}\left(\vec{r}, \vec{r}_{0}\right)\right] .
$$

Here, $\widetilde{p}\left(\vec{r}_{0}, k\right)$ represents the spectrum of the pressure $p\left(\vec{r}_{0}, \bar{t}\right)$ detected at $\vec{r}_{0}$ on $S ; *$ denotes complex conjugate; $\nabla_{0}$ denotes the gradient over variable $\vec{r}_{0} ; \hat{n}_{0}^{s}$ denotes the normal vector of surface $S$ pointing inward; the term in the square brackets represents dipole radiation; $\widetilde{G}_{k}^{(o u t)}\left(\vec{r}, \vec{r}_{0}\right)$ is a Green's function representing a monochromatic outgoing spherical wave:

$$
\widetilde{G}_{k}^{(o u t)}\left(\vec{r}, \vec{r}_{0}\right)=\exp \left(i k\left|\vec{r}^{\prime}-\vec{r}_{0}\right|\right) /\left(4 \pi\left|\vec{r}^{\prime}-\vec{r}_{0}\right|\right) .
$$

\section{BOUNDARY CONDITIONS}


When an acoustic wave encounters an interface between two media of different acoustic impedances, the boundary conditions require that both the pressure and the normal medium velocity (velocity component perpendicular to the interface) be continuous, where the acoustic characteristic impedance $(Z)$ is defined by $Z=\rho v_{s}$. The pressure continuity (boundary condition I) can be considered as a consequence of the conservation of energy and the normal velocity continuity (boundary condition II) as a consequence of the conservation of mass.

In the case where a plane wave propagating in medium 1 of impedance $Z_{1}$ is normally incident upon a planar interface with medium 2 of $Z_{2}$, the pressure reflection coefficient can be calculated by

$$
r_{n}=\frac{Z_{2}-Z_{1}}{Z_{2}+Z_{1}} .
$$

At a hard boundary, the acoustic impedance in the incident medium is much less than that in the transmitted medium, i.e., $Z_{1}<<Z_{2}$, leading to $r_{a}=1$. In this case, boundary condition II is zero (zero normal velocity or zero normal pressure gradient), which is mathematically referred to as a homogeneous Neumann boundary condition, and the incident pressure is reflected in equal amplitude with no sign change. Acoustic wave reflection in soft tissue by an interface with metal can be modeled approximately by a hard boundary. For example, $r_{n}=0.94$ for a water-steel interface, where the acoustic impedance of water is close to that of soft biological tissue.

At a soft boundary, the acoustic impedance in the incident medium is much greater than that in the transmitted medium, i.e., $Z_{1} \gg Z_{2}$, leading to $r_{a}=-1$. In this case, boundary condition I is zero (zero pressure), which is mathematically referred to as a homogeneous Dirichlet boundary condition, and the incident pressure is reflected in equal amplitude with a sign change. Acoustic wave reflection in soft tissue by an interface with air can be modeled well by a soft boundary. For example, $r_{n}=-0.9994$ for a water-air interface.

We can use the following algorithm to reconstruct the image:

1. Image the original detectors about the planar boundary from $\vec{r}_{0}$ to $\vec{r}_{0}^{*}$.

2. Assign the received photoacoustic signals from the original detectors to the corresponding image detectors with no sign change for hard boundary: $\tilde{p}\left(\vec{r}_{0}^{*}, k\right)=\widetilde{p}\left(\vec{r}_{0}, k\right)$, or with sign change for soft boundary: $\tilde{p}\left(\vec{r}_{0}^{*}, k\right)=-\tilde{p}\left(\vec{r}_{0}, k\right)$. Of course, we also have $p\left(\vec{r}_{0}^{*}, t\right)=p\left(\vec{r}_{0}, t\right)$.

3. Apply the reconstruction algorithm in Eq. (6) for an infinite medium to this "imaged" infinite medium, where the detection surface includes both the original and the image detectors.

\section{RESULTS}

\subsection{Simulation Results}

We simulated two-dimensional planar imaging of a medium that has either hard or soft boundaries $(Z=1$ or -1$)$. The buried object is a triangle that consists of four optically absorbing lines [see Fig. 1], which represent four typical orientations of line objects - parallel, perpendicular, and oblique to a boundary. We can write the detected signal from the object as follows:

$$
p_{m}=\frac{1}{r} \int_{L} I\left(t-r / c_{0}\right) d l,
$$


where $I(t)$ denotes the impulse response of the ultrasonic detector to a point object, $L$ denotes the object, $c_{0}$ denotes the speed of sound in the propagation medium, and $r$ denotes the distance between the detector and a small differential element $d l$ on the object.

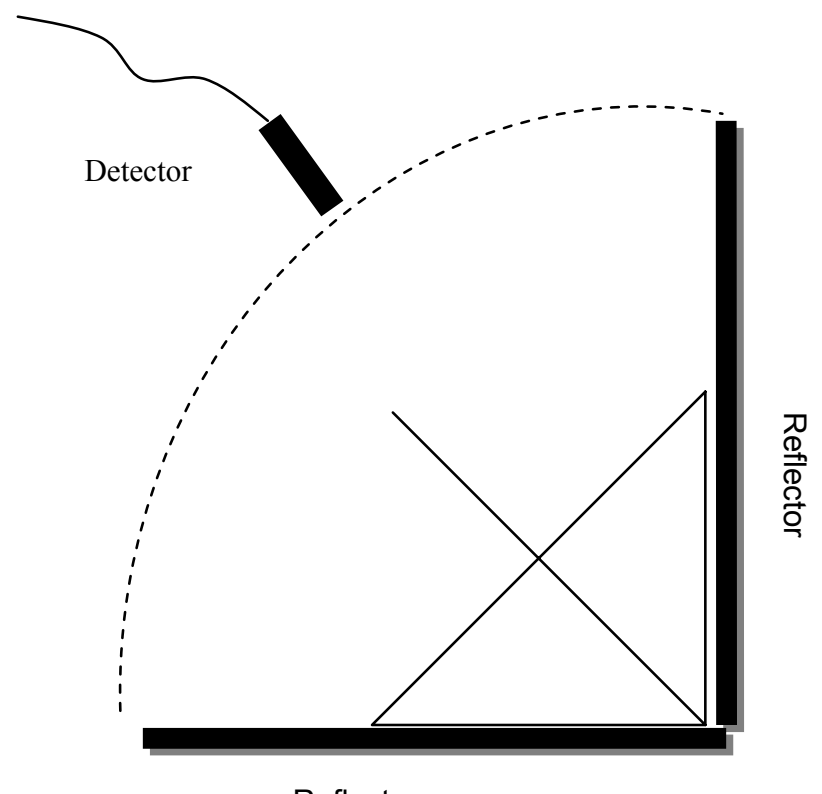

Reflector

Fig. 1 Schematic of the numerical scanning

a)
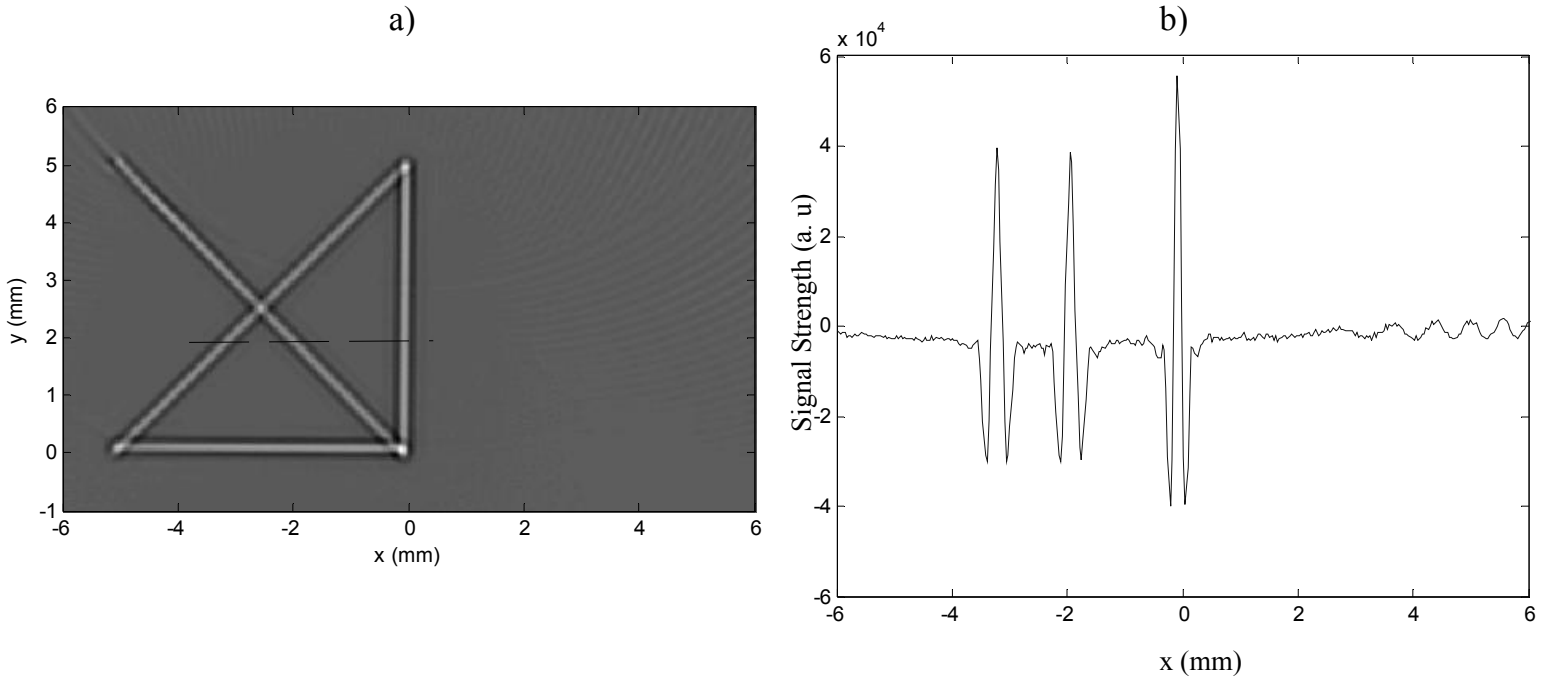

Fig. 2 (a) simulated reconstructed image after a $2 \pi$ circular scan; (b) the signal profile corresponding to the location indicated by the dash line in (a)

In our numerical simulations, we choose [9] 


$$
I(t)=A t^{-3} \cos \left(2 \pi f_{0} t\right) e^{-K f_{0} t},
$$

where $A$ is a constant, and $f_{0}$ is the center frequency. With $K=3.833$, this function gives a bandwidth of $80 \%$. In our simulation, $f_{0}$ is set to $6 \mathrm{MHz}$. In addition, an additive white Gaussian noise is imposed to the signal.

Fig. 1 shows the schematic of the scanning we used in our numerical simulation. The object consists of four lines, and two reflectors are located on the left side and the bottom. Because of the existence of the reflectors, the detector can only scan $\pi / 2$ angle, instead of $2 \pi$. Fig. 2(a) shows an image reconstructed with simulated data from a $2 \pi$ (fullcircular) scan in the case that reflectors are removed. Fig. 2(b) shows the one-dimensional (1D) distribution of image amplitude along the dashed line in Fig. 2(a). Fig. 3 shows the simulation result when hard planar boundaries $(Z=1)$ are placed at $x=0$ and $y=0$. Fig. 3(a) shows the image reconstructed with simulated data from a $\pi / 2$ (quarter-circular) scan using the conventional algorithm. Fig. 3(b) shows the image reconstructed with the same data using the new algorithm proposed in this paper. In both Figs. 3(a) and (b), the mirrored images of the buried triangular object are clearly visible. Fig. 3(c) shows the 1D distribution of image amplitude along the dashed line in Fig. 3(a) and (b). Compared with the result for the full-circular scan shown in Fig. 2(b), an extra half cycle is present at the end of every pulse at the data without applying the new algorithm, which deteriorates the spatial resolution by causing a "shadow" in the reconstructed image. In Fig. 3(a), shadows and streaks appear area owing to the limited view; in addition, the mirror image of the horizontal line is not recovered. By implementing the new reconstruction algorithm described in Sec. 3, we eliminate the above artifacts as shown in Fig. 3(b). The 1D distribution of image amplitude along the dashed line in Fig. 3(b) is presented in Fig. 3(c), which shows that the extra half cycle disappears. Also, one line is missing on Fig. 3(a) and is recovered in Fig. 3(b) after applying the new algorithm. Fig. 4 shows similar results as Fig. 3. The medium, however, has soft boundaries $(\mathrm{Z}=-1)$. As a result, the mirror image has an opposite polarity.

It is also obvious that the new algorithm increases the image amplitude, which improves the SNR of the images. For example, without applying new algorithm, the SNRs for the peaks on Fig. 3(c) and Fig. 4(c) (solid lines) are $32 \mathrm{~dB}$ and $35 \mathrm{~dB}$, respectively; by contrast, after applying the new algorithm, the corresponding SNRs in Fig. 3(c) and Fig. 4(c) (dash lines) are $35 \mathrm{~dB}$ and $38 \mathrm{~dB}$, respectively. Therefore, a 3-dB improvement is achieved in both cases.

\subsection{Experimental Results}

In the experiment, a virtual ultrasound point detector developed in our lab was used for photoacoustic detection [10]. The virtual point detector has a center frequency around $6 \mathrm{MHz}$. The buried object was made of two human hairs with a $\sim 40 \mu \mathrm{m}$ diameter and a cross shape was used. A glass plate served as a hard planar boundary, whereas a piece of white plastic foam served as a soft planar boundary. The boundary was placed next to one of the left side of the buried object. Water was used as the acoustic coupling medium. The reconstructed data are presented in Figs. 5 - 7. Fig. 5(a) shows the reconstructed image from a $2 \pi$ scan of the buried object in a boundary-free medium. The $1 \mathrm{D}$ distribution of image amplitude along the dashed line in Fig. 5(a) is plotted in Fig. 5(b). Figs. 6 and 7 show the reconstructed images from data acquired under the hard and soft boundary conditions, respectively. The boundary was located at the position $\mathrm{x}=0$. Only one boundary was present. Because of the boundary, the detector can only perform a $\pi$ scan (semi-circular) in our experiments. Fig. 6(a) shows the image reconstructed from a $\pi$ scan using the conventional reconstruction algorithm. The experimental results show heavy 'shadows' on the left side of the image when without applying the new algorithm. At the same time, the image is deformed compared to Fig. 5. After applying the new algorithm in Fig. 6(b), the shadow is reduced, and more important, the image is not deformed any more, and the image quality is improved. In addition, the image amplitude is increased by the new algorithm. For example, the SNR of the left peak is increased from $25 \mathrm{~dB}$ to $30 \mathrm{~dB}$ in the hard boundary case and from $26 \mathrm{~dB}$ to $30 \mathrm{~dB}$ in the soft boundary case. The 1D distributions of images amplitude along the dashed line are plotted in Fig. 6(c). Fig. 7 shows the similar results as Fig. 6 for a soft boundary condition. 

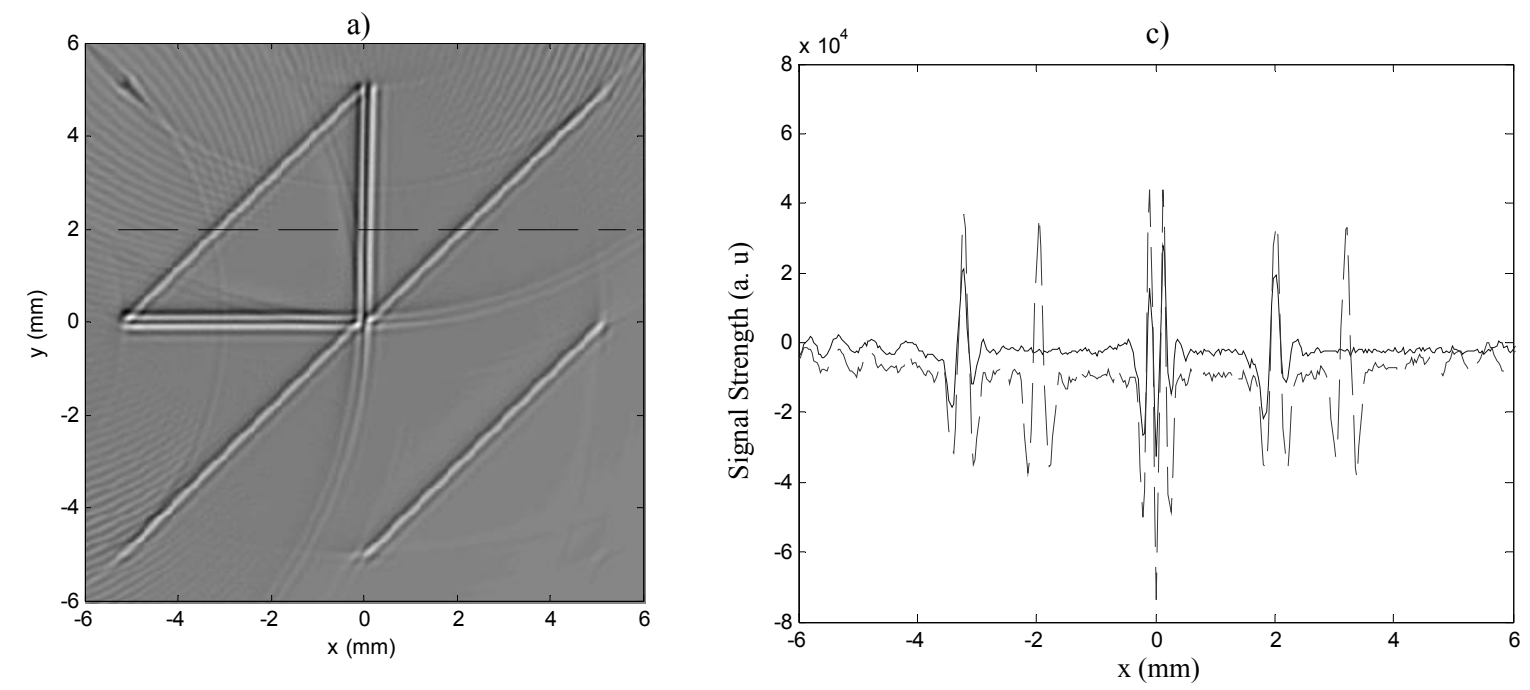

b)

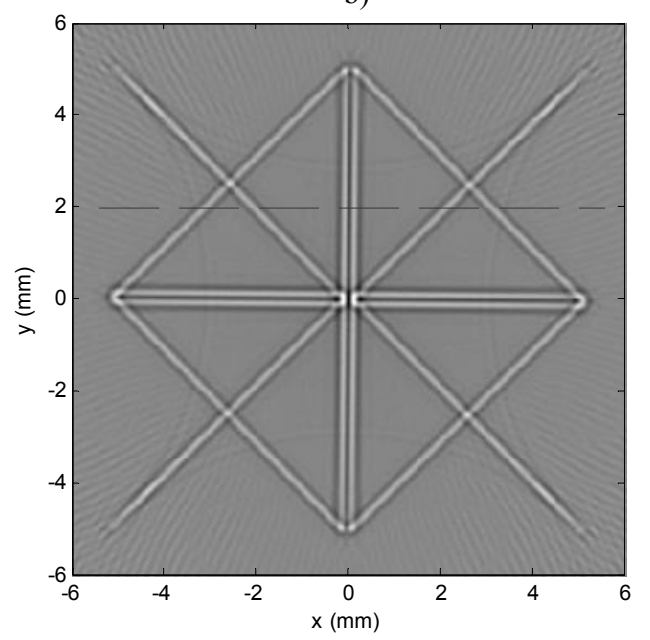

Fig 3. Simulated results. Hard boundaries are placed at $\mathrm{x}=0$ and $\mathrm{y}=0 \mathrm{~mm}$. (a) simulated reconstructed image with data from a $\pi / 2$ semi-circular scan; (b) simulated reconstructed image with the new reconstruction algorithm using the same data as (a); (c) the signal profile corresponding to the location indicated by the dash line in (a) and (b), solid line is from (a) and dash line is from (b). 
a)

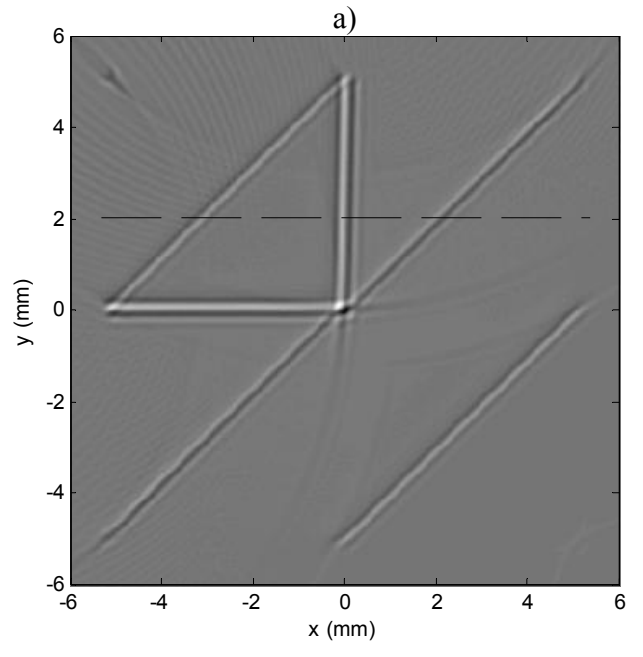

b)

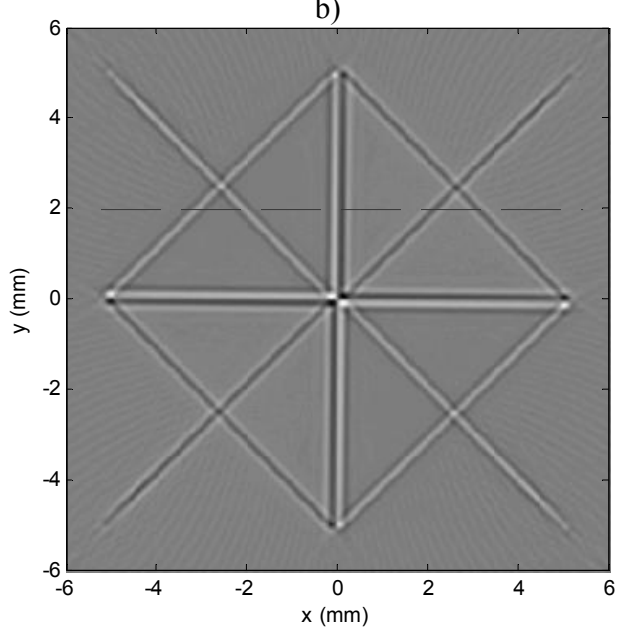

c)

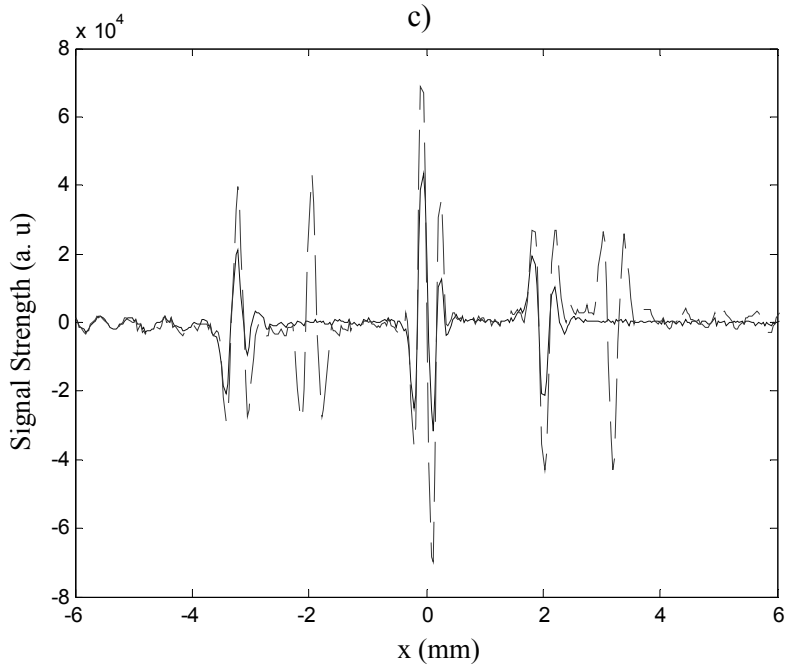

Fig 4. Simulated results. Soft boundaries are placed at $\mathrm{x}=0$ and $\mathrm{y}=0 \mathrm{~mm}$. (a) simulated reconstructed image with data from a $\pi / 2$ semi-circular scan; (b) simulated reconstructed image with the new reconstruction algorithm using the same data as (a); (c) the signal profile corresponding to the location indicated by the dash line in (a) and (b), solid line is from (a) and dash line is from (b).

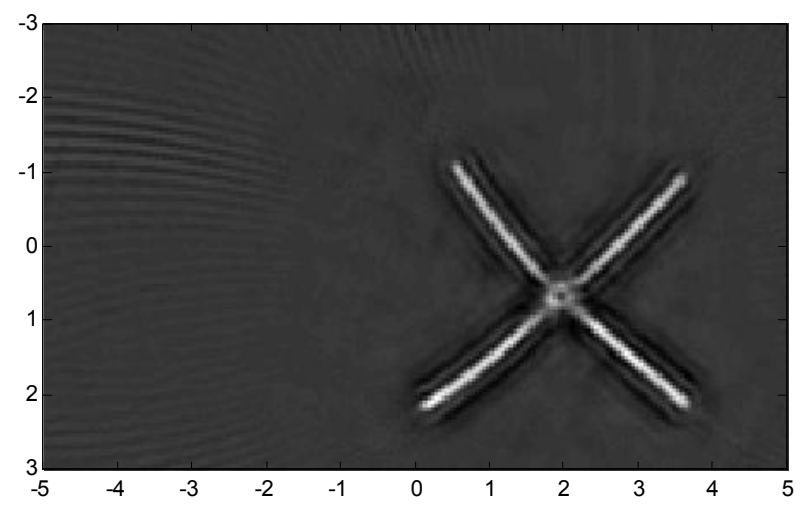

Fig. 5 The reconstructed image after a boundary-free $2 \pi$ circular scan. 
a)

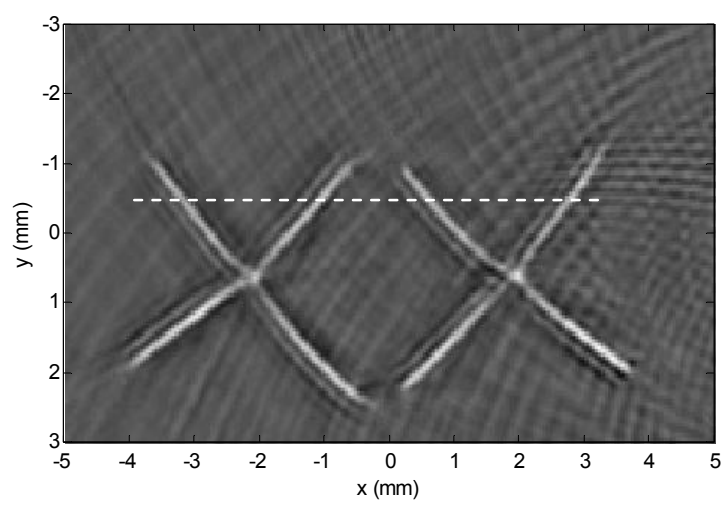

Fig. 6 Experimental results. A hard boundary is placed near $\mathrm{x}=0 \mathrm{~mm}$. (a) reconstructed image with data from a $\pi$ semi-circular scan; (b) reconstructed image with the new reconstruction algorithm using the same data as (a); (c) the signal profile corresponding to the location indicated by the dash line in (a) and (b); Solid line is from (a) and dash line is from (b). b)

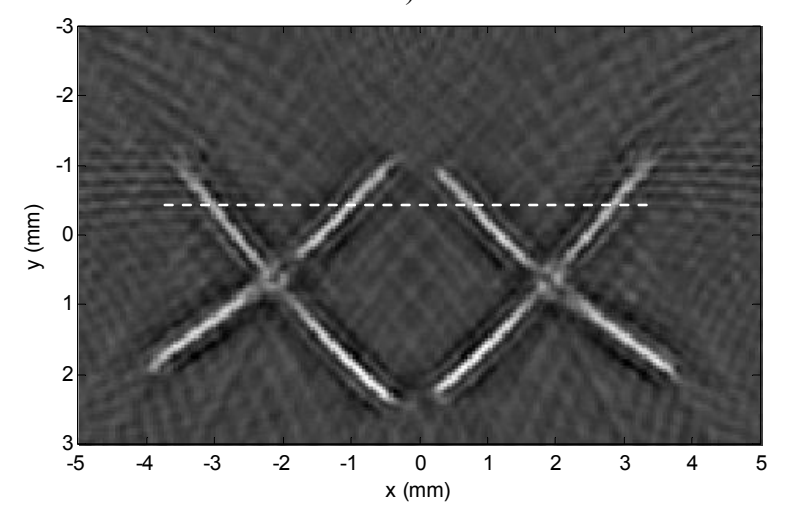

c)

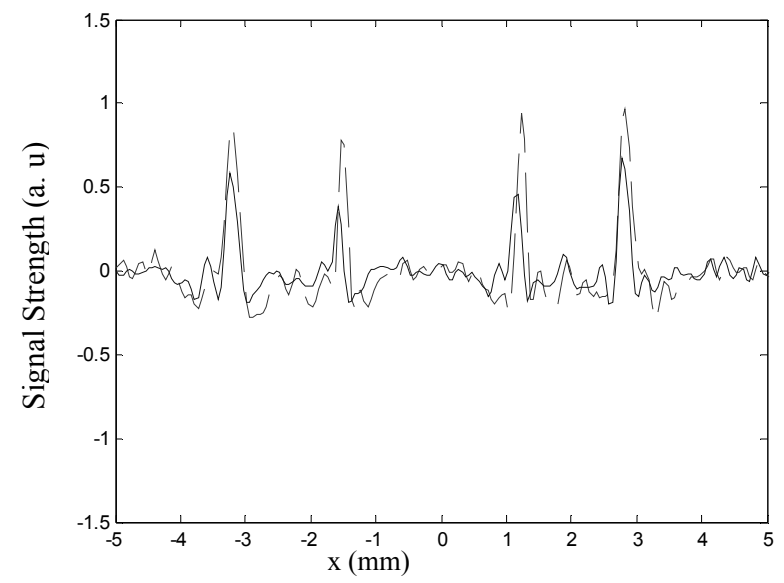


a)

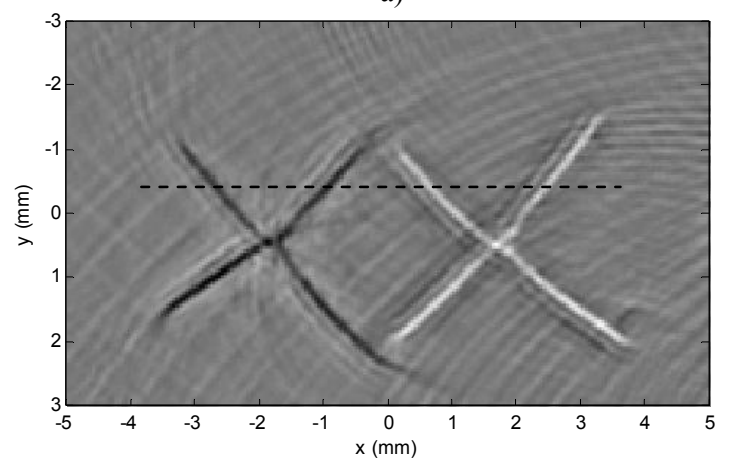

Fig. 7 Experimental results. A soft boundary is placed near $\mathrm{x}=0 \mathrm{~mm}$. (a) reconstructed image with data from a $\pi$ semi-circular scan; (b) reconstructed image with the new reconstruction algorithm using the same data as (a); (c) the signal profile corresponding to the location indicated by the dash line in (a) and (b); solid line is from (a) and dash line is from (b). b)

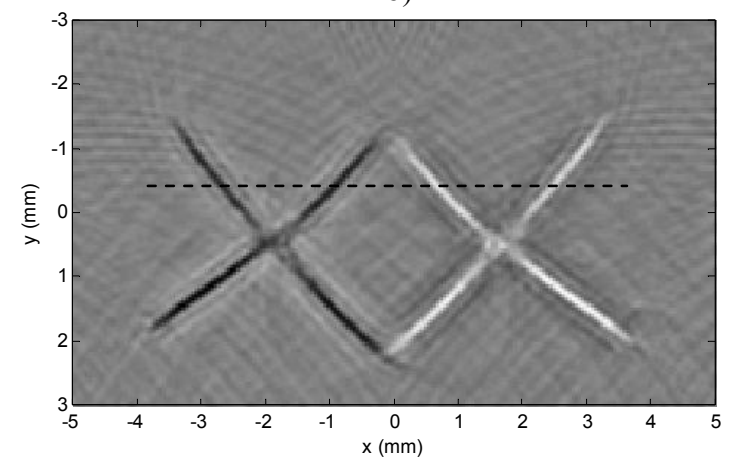

c)

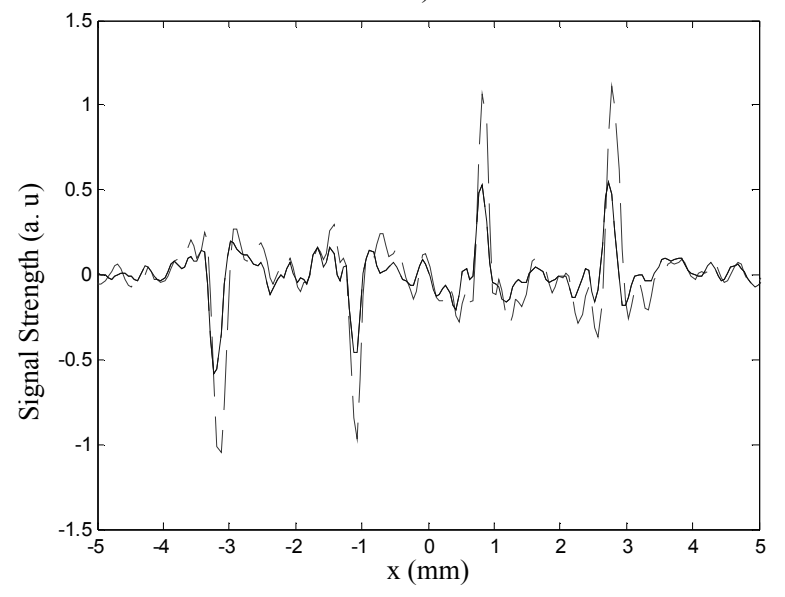

\section{SUMMARY}

In summary, the boundary conditions that must be considered in certain imaging configurations in PAT and the associated inverse solutions for image reconstruction are presented and validated. Partial planar with a planar boundary are considered, where the boundary can be either hard or soft. Analogously to the method of images of sources used for forward problems, the ultrasonic detectors are imaged about the boundary to satisfy the boundary condition for the inverse problem. The final surfaces that include both the original and the imaged detection surfaces form a completely enclosing surface in an infinite medium. The exact algorithms that have been developed for infinite media can be applied subsequently. Consequently, this new algorithm improves the quality of reconstructed images, compared with the limited-view reconstruction algorithms. The conclusions that are drawn here are equally applicable to thermoacoustic tomography, where radiofrequency, rather than optical, excitation is employed.

\section{ACKNOWLEDGEMENTS}

This project is sponsored by National Institutes of Health grants R01 EB000712 and R01 NS46214. 
1. K. P. Kostli, D. Frauchiger, J. J. Niederhauser, G. Paltauf, H. P. Weber, and M. Frenz, "Optoacoustic imaging using a three-dimensional reconstruction algorithm," IEEE Journal of Selected Topics in Quantum Electronics 7, 918-923 (2001).

2. Y. Xu, D. Z. Feng, and L. V. Wang, "Exact frequency-domain reconstruction for thermoacoustic tomography I: Planar geometry," IEEE Transactions on Medical Imaging 21, 823-828 (2002).

3. Y. Xu, M.-H. Xu, and L. V. Wang, "Exact frequency-domain reconstruction for thermoacoustic tomography II: Cylindrical geometry," IEEE Transactions on Medical Imaging 21, 829-833 (2002).

4. M.-H. Xu and L. V. Wang, "Time-domain reconstruction for thermoacoustic tomography in a spherical geometry," IEEE Transactions on Medical Imaging 21, 814-822 (2002).

5. D. Finch, S. K. Patch, and Rakesh, "Determining a function from its mean values over a family of spheres," SIAM Journal on Mathematical Analysis 35, 1213-1240 (2003).

6. M.-H. Xu and L. V. Wang, "Universal back-projection algorithm for photoacoustic computed tomography," Physical Review E 71, 016706 (2005).

7. M.-H. Xu and L. V. Wang, "Photoacoustic imaging in biomedicine," Review of Scientific Instruments 77, 041101 (2006).

8 M. Xu, and L. V. Wang, "Photoacoustic imaging in biomedicine," Rev. Sci. Instrum. 77, 041101, 1-22, (2006).

9. J. L. San Emeterio and L. G. Ullate, "Diffraction impulse response of rectangular transducers," Journal of Acoustic Society of America 92, 651-662 (1992).

10. Unpublished results. 